\title{
An Evolutionary Algorithm Based on a New Decomposition Scheme for Nonlinear Bilevel Programming Problems
}

\author{
Hecheng LI $^{1,2}$, Yuping WANG ${ }^{1}$ \\ ${ }^{I}$ School of Computer Science and Technology, Xidian University, Xi' an, China \\ ${ }^{2}$ Department of Mathematics and Information Science, Qinghai Normal University, Xining, China \\ E-mail: lihecheng@qhnu.edu.cn \\ Received July 24, 2009; revised September 21, 2009; accepted November 4, 2009
}

\begin{abstract}
In this paper, we focus on a class of nonlinear bilevel programming problems where the follower's objective is a function of the linear expression of all variables, and the follower's constraint functions are convex with respect to the follower's variables. First, based on the features of the follower's problem, we give a new decomposition scheme by which the follower's optimal solution can be obtained easily. Then, to solve efficiently this class of problems by using evolutionary algorithm, novel evolutionary operators are designed by considering the best individuals and the diversity of individuals in the populations. Finally, based on these techniques, a new evolutionary algorithm is proposed. The numerical results on 20 test problems illustrate that the proposed algorithm is efficient and stable.
\end{abstract}

Keywords: Nonlinear Bilevel Programming, Decomposition Scheme, Evolutionary Algorithm, Optimal Solutions

\section{Introduction}

The bilevel programming problem (BLPP) involves two optimization problems at different levels, in which the feasible region of one optimization problem (leader's problem/upper level problem) is implicitly determined by the other (follower's problem/lower level problem). The general bilevel programming problem can be formulated as follows.

$$
\left\{\begin{array}{l}
\min _{x \in X} F(x, y) \\
\text { s.t. } \quad G(x, y) \leq 0 \\
\min _{y \in Y} f(x, y) \\
\text { s.t. } \quad g(x, y) \leq 0
\end{array}\right.
$$

where $x \in R^{n}$ and $y \in R^{m}$ are called the leader's and follower's variables, respectively, $F(f): R^{n} \times R^{m} \rightarrow R$ is called the leader's (follower's) objective function, and the vector-valued functions $G: R^{n} \times R^{m} \rightarrow R^{p}$ and $g$ : $R^{n} \times R^{m} \rightarrow R^{q}$ are called the leader's and follower's constraints, respectively; the sets $X$ and $Y$ place additional constraints on the variables, such as upper and lower bounds or integrality requirements etc. This mathematical programming model arises when two in- dependent decision makers, ordered within a hierarchical structure, have conflicting objectives, and each decision maker seeks to optimize his/her objective function. In (1), the leader moves first by choosing a vector $x \in X \subseteq R^{n}$ in an attempt to optimize his/her objective function $F(x$, $y)$; the leader's choice of strategies affects both the follower's objective and decision space. The follower observes the leader's choice and reacts by selecting a vector $y \in Y \subseteq R^{m}$ that optimizes his/her objective function $f(x ; y)$. In doing so, the follower affects the leader's outcome.

BLPP is widely used to lots of fields such as economy, control, engineering and management [1,2], and more and more practical problems can be formulated as the bilevel programming models, so it is important to design all types of effective algorithms to solve different types of BLPPs. But due to its nested structure, BLPP is intrinsically difficult. It has been reported that BLPP is strongly NP-hard [2]. When all functions involved are linear, BLPP is called linear bilevel programming. It is the simplest one among the family of BLPPs, and the optimal solutions can occurs at vertices of feasible region. Based on these properties, lots of algorithms are proposed to solve this kind of problems [2-5]. When the functions involve nonlinear terms, the corresponding 
problem is called nonlinear BLPP, which is more complex and challenging than linear one. In order to develop efficient algorithms, most algorithmic research to date has focused on some special cases of bilevel programs with convex/concave functions[1,2,6-9], especially, convex follower's functions [10-14]. In these algorithms, the convexity of functions guarantees that the globally optimal solutions can be obtained. Moreover, some intelligent algorithms, such as evolutionary algorithms (EAs)/ genetic algorithms (GAs) [10,15-17], tuba search approaches [18], etc, have been applied to obtain the optimal solutions to multi-modal functions. However, a question arises when the follower's objective function is neither convex nor concave. On the one hand, it is difficult for us to obtain the globally optimal solution by using traditional optimization techniques based on gradients; on the other hand, if an intelligent algorithm, such as GA, is used to solve the follower's problem, it can cause too large amount of computation [16]. To the best knowledge of the authors, there are not any efficient algorithm for this kind of BLPPs.

In this paper, we consider a special class of nonlinear BLPP with nonconvex objective functions, in which the follower's objective is a function of the linear expression of all variables, and the follower's constraints are convex with respect to the follower's variables. There are no restrictions of convexity and differentiability on both the leader's and the follower's objective functions, which makes the model different from those proposed in the open literature. In view of the nonconvexity of the leader's objective, we develop an evolutionary algorithm to solve the problem. First, based on the structural features of the follower's objective, we give a new decomposition scheme by which the (approximate) optimal solution $y$ to the follower's problem can be obtained in a finite number of iterations. At the same time, the populations are composed of such points $(x, y)$ satisfying the follower's optimization problem, that is, $y$ is an optimal solution of the follower's problem when $x$ is fixed, which improves the feasibility of individuals. Then, to improve the efficiency of proposed algorithm, the better individuals than crossover parents are employed to design new crossover operator, which is helpful to generate better offspring of crossover. Moreover, we design a singleside mutation operator, which can guarantee the diversity of individual in the process of evolution.

This paper is organized as follows. The model of the bilevel programming problem and some conceptions are presented in Section 2, and a solution method of the follower's problem is given based on a decomposition scheme in Section 3. Section 4 presents an evolutionary algorithm in which new evolutionary operators are designed. Experimental results and comparison are presented in Section 5. We finally conclude our paper in Section 6 .

\section{Bilevel Programming Problem and Basic Notations}

Let us consider the bilevel programming problem defined as follows

$$
\left\{\begin{array}{l}
\min _{x \in X} F(x, y) \\
\text { s.t. } \quad G(x, y) \leq 0 \\
\min _{y \in Y} f(x, y)=\phi(B(x) y) \\
\text { s.t. } \quad g(x, y) \leq 0
\end{array}\right.
$$

where $g(x, y)$ is convex and twice continuously differential in $y$, the function $\varphi($.$) is continuous, B(x)=$ $\left(b_{1}(x), b_{2}(x), \ldots, b_{m}(x)\right)$ is vector-valued, where $b_{i}: R^{n}$ $\rightarrow R \quad i=1,2, \ldots, m \quad$ and $\quad X=\left\{\left(x_{1}, \ldots, x_{n}\right)^{T} \in R^{n} \mid\right.$ $\left.l_{i} \leq x_{i} \leq u_{i}, i=1, \ldots, n\right\} \quad, \quad Y=\left\{\left(y_{1}, \ldots, y_{m}\right)^{T} \in R^{m} \mid \quad \bar{l}_{j} \leq\right.$ $\left.y_{j} \leq \bar{u}_{j}, j=1, \ldots, m\right\} \quad l_{i}, u_{i}, \bar{l}_{j}, \bar{u}_{j}, i=1,2, \ldots n, j=1,2, \ldots, m$, are all real constant. Now we introduce some related definitions and notations [2] as follows.

1) Search space: $\Omega=\{(x, y) \mid x \in X, y \in Y\}$;

2) Constraint region:

$$
S=\{(x, y) \in \Omega \mid G(x, y) \leq 0, g(x, y) \leq 0\} ;
$$

3) For $x$ fixed, the feasible region of follower's problem:

$$
S(x)=\{y \in Y \mid g(x, y) \leq 0\}
$$

4) Projection of $S$ onto the leader's decision space:

$$
S(X)=\{x \in X \mid \exists y, \text { such that }(x, y) \in S\}
$$

5) For each $x \in S(X)$, the follower's rational reaction set:

$$
M(x)=\{y \in Y \mid y \in \arg \min \{f(x, v), v \in S(x)\}\}
$$

6) Inducible region:

$$
I R=\{(x, y) \in S \mid y \in M(x)\} .
$$

In terms of aforementioned definitions, (2) can also be written as:

$$
\min \{F(x, y),(x, y) \in I R\}
$$

In order to ensure that (2) is well posed, in the remainder, we always assume that:

A1: $S$ is nonempty and compact;

A2: For all decisions taken by the leader, each follower has some room to react, that is, $S(x) \neq \varphi$.

A3: The follower's problem has at most finite optimal solutions for each fixed $x$.

Since the leader's functions may be nonconvex or nondifferential in (2), we use an evolutionary algorithm to solve the problem. However, due to the nested structure, the problem (2) always has a narrow inducible re- 
gion (feasible region). If the individuals $(x, y)$ are generated at random, there are large numbers of infeasible points in the populations, which makes the efficiency of EA decreased. To overcome the disadvantage, for $x$ fixed, we first solve the follower's problem to get the optimal solution $y$. After doing so, the point $(x, y)$ at least satisfies or approximately satisfies the follower's optimization. But the follower's optimization problem may be nonconvex, how one can easily obtain the follower's globally optimal solutions $y$ for a fixed $x$ ? We finish the step in the next section

\section{Solution Method of the Follower's Problem}

For any fixed $x$, we consider the follower's problem of (2), that is,

$$
\left\{\begin{array}{l}
\min _{y \in Y} f(x, y)=\phi(B(x) y) \\
\text { s.t. } g(x, y) \leq 0
\end{array}\right.
$$

The optimal solutions to (3) can be found in several steps given as follows. Firstly, we solve the following nonlinear convex programming problems with linear objective function.

$$
\left\{\begin{array}{l}
\min _{y \in Y} B(x) y \\
\text { s.t. } \quad g(x, y) \leq 0
\end{array}\right.
$$

and

$$
\left\{\begin{array}{cl}
\min _{y \in Y} \quad-B(x) y \\
\text { s.t. } & g(x, y) \leq 0
\end{array}\right.
$$

here, both (4) and (5) are convex programming problems, as a result, the optimal solutions can be obtained easily by the existing algorithms. We denote by $y_{\min }$ and $y_{\max }$ the optimal solutions to (4) and (5), respectively; setting $B(x) y_{\min }=a$ and $B(x) y_{\max }=b$. In fact, we only need to consider the function $\varphi$ over the interval $[a, b]$. Secondly, let us find the monotone intervals of the function $\varphi$ in $[a, b]$, without any loss of generality, denote these intervals by $\left[a_{i}, b_{i}\right] ; i=1,2, \ldots, k$, and the index set corresponding to all increasing intervals is denoted by $I_{1}$, while that corresponding to all decreasing intervals by $I_{2}$. Thirdly, we consider the families of nonlinear programming problems as follows.

$$
\left\{\begin{array}{l}
\min _{y \in Y} B(x) y \\
\text { s.t. } \quad g(x, y) \leq 0, \\
a_{i} \leq B(x) y \leq b_{i}
\end{array} \quad i \in I_{1}\right.
$$

and

$$
\left\{\begin{array}{l}
\min _{y \in Y}-B(x) y \\
\text { s.t. } \quad g(x, y) \leq 0, \\
a_{i} \leq B(x) y \leq b_{i}
\end{array} \quad i \in I_{2}\right.
$$

Evidently, the optimal solution to (3) can be obtained by solving (6) and (7). But it is computationally expensive to solve all of these nonlinear programming problems given by (6) and (7). In fact, we have

Theorem 1. Each among (6) and (7) can get its minimum on the hyperplanes presented by $\mathrm{B}(\mathrm{x}) \mathrm{y}=\pi_{i},\left(\pi_{i}\right.$ $=\mathrm{a}_{\mathrm{i}}$ or $\left.\mathrm{b}_{\mathrm{i}}, \mathrm{i}=1,2, \ldots, \mathrm{k}\right)$.

Proof. Note that $B(x) y$ is linear with respect to $y$, as a result, it can achieve the minimum and maximum values at the boundary of $S(x)$, and the two points were denoted by $y_{\min }$ and $y_{\max }$. Let us recall that $S(x)$ is convex, it follows that each point on the line segment $y_{\min } y_{\max }$ is in $S(x)$. Further, $B(x) y$ is continuous in the compact set $y_{\min } y_{\max }$, it means for each $\pi_{i} \in[a, b]$, there exists a point $y_{\pi_{i}}$ on $y_{\min } y_{\max }$ such that $B(x) y_{\pi_{i}}=\pi_{i}$. As a result, it is easy to see that for each optimization problem presented by (6) and (7), there is a point satisfying $B(x) y=$ $\pi_{i}$ in the feasible region. This completes the proof.

Since $f(x, y)=\varphi(B(x) y)$ is constant over each hyperplane, we select a hyperplane on which $f(x, y)$ has the smallest value. Recall assumption $A 3$, there exists only a feasible point in the hyperplane. But how can one easily take the point from the hyperplane? Let us finish the process in the last step. Finally, draw a line segment connecting points $y_{\min }$ and $y_{\max }$, which can be presented by the expression with a parameter $t: y=y_{\min }+t\left(y_{\max }-\right.$ $\left.y_{\min }\right)$. One can obtain easily a feasible point on the hyperplane by considering both the expression and the equation of the hyperplane. If there are more than one optimal solution, that is, there exist several hyperplanes on which $f(x, y)$ has the same smallest value, in terms of $A 3$, the number of feasible points is finite in these hyperplanes, we can select one among them according to the fitness function values given in next section. The procedure of solving the follower's problem is shown in Figure 1. In Figure 1, the elliptical region is the feasible region of the follower's problem for some fixed $x$. In terms of the

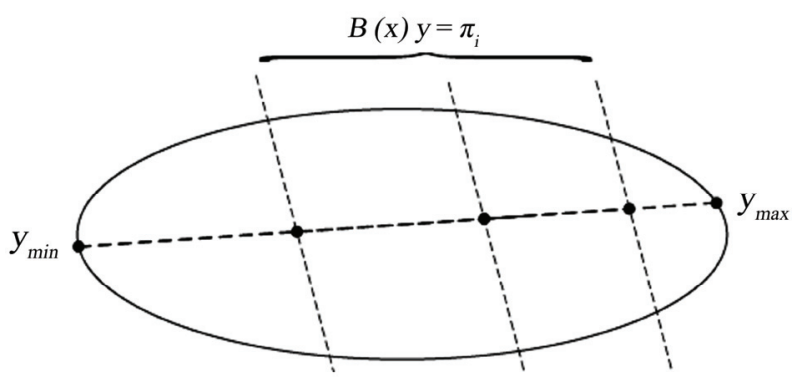

Figure 1. Follower's feasible region and hyperplanes used to obtain the optimal value of $\varphi$. 
monotonicity of $\varphi$, one can give the hyperplanes $B(x) y=\pi_{i}$, and $f(x, y)$ can achieve the optimal value on one or some hyperplanes. Also, $\varphi$ is constant on each hyperplane, it means that the optimal solution of the follower's problem can be found by checking the values of $\varphi$ at the black points on $y_{\min } y_{\max }$.

\section{Proposed Algorithm}

In the section, we begin with the fitness function, crossover and mutation operators, and then propose an evolutionary algorithm with real number encoding to solve (2).

\subsection{Fitness Function}

Let us apply the penalty method to deal with the leader's constraints. In order to use the technique efficiently, we design a dynamic parameter inspired by the sequential weight increasing factor technique (SWIFT) proposed by B. V. Sheela and P. Ramamoorthy [19]. The fitness function is denoted by $R(x, y)=F(x, y)+M_{\mathrm{g}}$ $\max \left\{0, G_{i}(x, y), i=1, \ldots, p\right\}$. Where $g$ stands for the generation number, $G_{i}(x, y)$ are the components of $G(x, y)$; $M_{g}$ are penalty parameters (where $M_{1}$ is given in advance as a positive constant, $g=1,2, \ldots)$ and computed as follows.

Choose $n+1$ best points $p_{0}, p_{1}, \ldots, p_{n}$ from the current population. Let

$$
d=\frac{1}{n+1} \sum_{i=0}^{n}\left\|p_{i}-p_{G}\right\|
$$

Here $p_{G}=\frac{1}{n+1} \sum_{i=0}^{n} p_{i}$, and $M_{g}$ is updated by the following formula.

$$
M_{g+1}=\max \left\{M_{g}, \frac{1}{d}\right\}
$$

While $n+1$ points chosen as above are affine independent, these points constitute a simplex $\left\{p_{0}, p_{1}, p_{2}\right.$, $\left.\ldots, p_{n}\right\}$; and $M_{g} \rightarrow \infty$ as the generation number $g$ increases.

\subsection{Crossover Operator}

Let $\mathrm{N}$ denote the population size. Rank all points in the population pop according to the fitness values from small to large. Without any loss of generality, we also denote these points by $p_{1}, p_{2}, \ldots, p_{N}$, and let $R_{i}>0$ stand for the fitness values of $p_{i}, \mathrm{i}=1,2, \ldots, \mathrm{N}$. We give the crossover operator as follows.

Let $p_{i}$ be a selected individual for crossover. The crossover offspring $o_{i}$ of the individual is got as follows.

$$
o_{i}= \begin{cases}p_{i}+r \delta\left(p_{G}^{i}-p_{i}\right), & i>1 \\ p_{i}+r \delta\left(p_{i}-p_{0}\right), & i=1\end{cases}
$$

where $r \in[0,1]$ is random, $\delta$ is a positive constant, $p_{0}$ is arbitrarily chosen in the current population and

$$
p_{G}^{i}=\sum_{l=1}^{i-1} \mu_{l} p_{l}
$$

$$
\mu_{l}=\frac{1 / R_{l}}{\sum_{j=1}^{i-1} 1 / R_{j}}, \quad l=1, \ldots, i-1
$$

In the designed crossover operator, all better individuals than the crossover parent are used to provide a good direction for crossover, and the coefficients $\mu_{l}$ make $p_{G}^{i}$ closer to the points with smaller fitness.

\subsection{Single-Side Mutation Operator}

Let $\hat{p}_{i}=\left(\hat{p}_{i}^{1}, \ldots, \hat{p}_{i}^{n}\right)$ be a parent individual for mutation. The mutation offspring $\hat{o}_{i}$ of $\hat{p}_{i}$ is generated as follows.

At first, to select a component $\hat{p}_{i}^{j}$ of $\hat{p}_{i}$ at random, then the hyperplane $\hat{p}^{j}=\hat{p}_{i}^{j}$ divides the search space $X$ into two hypercubes. Let $\Delta^{-}$represents the hypercube in which any point satisfies $\hat{p}^{j} \leq \hat{p}_{i}^{j}$ while $\Delta^{+}$stands for the other, $V\left(\Delta^{-}\right)\left(V\left(\Delta^{+}\right)\right)$presents the volume of $\Delta^{-}\left(\Delta^{+}\right)$; and pop $^{-}$represents the set of all individuals belonging to $\Delta^{-}$, pop $^{+}$stands for the set of other points in the population; $|W|$ presents the number of elements in the set $W$. Then

$$
\hat{o}_{i}= \begin{cases}\hat{o}_{i}^{1}, & \frac{\left|p o p^{-}\right|}{V\left(\Delta^{-}\right)+\mu} \leq \frac{\mid \text { pop }^{+} \mid}{V\left(\Delta^{+}\right)+\mu}, \\ \hat{o}_{i}^{2}, & \text { otherwise }\end{cases}
$$

where $\hat{o}_{i}^{1}\left(\hat{o}_{i}^{2}\right)$ is a point taken randomly in $\Delta^{-}\left(\Delta^{+}\right)$; $\mu$ is a small positive number, which keeps the denominators away from zero.

The mutation operator designed so is helpful to uniformly generate points in $X$, which can help to prevent the algorithm from the premature convergence.

\subsection{Evolutionary Algorithm Based on the New Decomposition Scheme (EABDS)}

Step 1. (Initialization) Randomly generate $N$ initial points $x_{i} \in X, i=1,2, \ldots, N$. For each $x_{i}$ fixed, we solve the follower's problem for $y_{i}$ via the algorithm presented in Section 3. All of the points $\left(x_{i}, y_{i}\right)$ form the initial population $p o p(0)$ with population size $N$. Let $k=0$; 
Step 2. (Fitness) Evaluate the fitness value $R(x, y)$ of each point in $\operatorname{pop}(k)$;

Step 3. (Crossover) Randomly select a parent $(x, y)$ from $\operatorname{pop}(k)$ according to the crossover probability $p_{c}$. We first execute the crossover for the component $x$ of the parent, and denote the offspring by $\hat{x}$. For fixed $\hat{x}$, the follower's problem is solved to get the optimal solution $\hat{y}$. Then we can obtain a crossover offspring $(\hat{x}, \hat{y})$. Let $O 1$ stand for the set of all these offspring;

Step 4. (Mutation) Randomly select parents from pop $(k)$ according to the mutation probability $p_{m}$. For each selected parent $(x, y)$, we first execute the mutation for $x$ and get its offspring $\bar{x}$, then for fixed $\bar{x}$, we optimize the follower's problem for $\bar{y} .(\bar{x}, \bar{y})$ is the mutation offspring of $(x, y)$. Let $O 2$ represent the set of all these offspring.

Step 5. (Selection) Let $O=O 1 \cup O 2$. We evaluate the fitness values of all points in $O$, select the best $N_{1}$ points from the set $\operatorname{pop}(k) \cup O$ and randomly select $N-N_{1}$ points from the remaining points of the set. These selected points form the next population pop $(k+1)$;

Step 6. If the termination condition is satisfied, then stop; otherwise, let $k=k+1$, go to Step 3 .

\section{Simulation}

We select 14 test problems from the references $[4,7,8,10$, 12-15,17]. All problems are nonlinear except for $g 01$, $g 02, g 05$, and $g 08$. In these problems, the function involved is differentiable and convex. In order to test the effectiveness of the proposed algorithm for nonconvex or nondifferentiable problems, we also construct 6 additional test problems as follows.

We first choose 2 test problems $g 10$ and g08 randomly from the 14 problems:

$$
\begin{aligned}
& \text { g10) }\left\{\begin{array}{l}
\min _{0 \leq x \leq 15} x^{2}+(y-10)^{2} \\
\text { s.t. } \quad-x+y \leq 0 \\
\min _{0 \leq y \leq 20}(x+2 y-30)^{2} \\
\text { s.t. } x+y \leq 0
\end{array}\right. \\
& \text { g08) }\left\{\begin{array}{l}
\min _{x \geq 0}-8 x_{1}-4 x_{2}+4 y_{1}-40 y_{2}-4 y_{3} \\
\min _{y \geq 0} x_{1}+2 x_{2}+y_{1}+y_{2}+2 y_{3} \\
s . t . \quad-y_{1}+y_{2}+y_{3} \leq 1 \\
2 x_{1}-y_{1}+2 y_{2}-0.5 y_{3} \leq 1 \\
2 x_{2}+2 y_{1}-y_{2}-0.5 y_{3} \leq 1
\end{array}\right.
\end{aligned}
$$

and replace the follower's objective functions of the two problems by new constructed ones, respectively. As a result, we get problems $g 15-g 20$, in which problems $g 15$ $g 17$ are the same as problem $g 10$ except for

g15) $f(x, y)=|x+2 y-30|$;

g16) $f(x, y)=\sin (x+2 y-30)$;

g17) $f(x, y)=\cos (x+2 y-30)$;

While problems $g 18-g 20$ have the same functions as problem $g 08$ except for

g18) $f(x, y)=\left|x_{1}+2 x_{2}+y_{1}+y_{2}+2 y_{3}\right|$

g19) $f(x, y)=\sin \left(x_{1}+2 x_{2}+y_{1}+y_{2}+2 y_{3}\right)$

g20) $f(x, y)=\cos \left(x_{1}+2 x_{2}+y_{1}+y_{2}+2 y_{3}\right)$

Generally speaking, the functions constructed so are nondifferentiable or nonconvex, which makes the corresponding BLPPs more difficult to solve than those with differentiable and convex objective functions.

The parameters are chosen as follows: the population size $N=30$, the crossover probability $p c=0.8$, the mutation probability $p_{m}=0.2, N_{1}=20, \delta=2$ and $M_{1}=100$. For $g 01-g 20$, the algorithm stops when the best result can not be improved in 20 successive generations or after 50 generations. We execute EABDS in 30 independent runs on each problem on a computer (Intel Pentium IV-2.66GHz), and record or compute the following data:

$1)$ Best values $\left(F_{\text {best }}\right)$, worst values $\left(F_{\text {worst }}\right)$, mean $\left(F_{\text {mean }}\right)$, median $\left(F_{\text {median }}\right)$ and standard deviation $(S t d)$ of the objective function values in all 30 runs.

2) Best solutions $\left(x^{*}, y^{*}\right)$ in all 30 runs.

3) Mean of generation numbers $\left(g_{\text {mean }}\right)$, and mean numbers of the individuals (MNI) used by EABDS.

All results are presented in Tables 1-2, in which Table 1 provides the comparison of the results found by both EABDS and the compared algorithms for problems g01-g20. Table 2 shows the best solutions found by both EABDS and the algorithms in the related references for $g 01-g 20$, and $g_{\text {mean }}$ and $C P U$ used by EABDS are provided in Table 2. NA means that the result is not available for the algorithms, '*' presents the problem is the maximization model and Ref stands for the related algorithms in references in Tables 1-2.

It can be seen from Table 1 that for problems $g 01, g 03$, $g 05, g 07[13,17], g 08$ [17], $g 09$ [15] and $g 10$, the best results found by EABDS are much better than those by the compared algorithms in the references, which indicates the algorithms in the related conferences can't find the globally optimal solutions of these problems. For the constructed problems g15-20, EABDS gives uniform results. From the constructed objective functions, one can easily see that $g 15$ has the same optimal solutions as $g 10$, and $g 18$ has the same optimal solutions as $g 08$, which is also illustrated by the computational results in Tables 1-2. This means that EABDS can be used to solve nonlinear BLPPs with the nondifferentiable and noncom- 
Table 1. Comparison of the best results found by EABDS and the algorithms presented by related references.

\begin{tabular}{|c|c|c|c|c|c|c|}
\hline \multirow{2}{*}{ No. } & \multicolumn{5}{|c|}{ EABDS-F $(x, y)$} & \multirow{2}{*}{$\frac{R e f}{F_{\text {best }}}$} \\
\hline & $F_{\text {best }}$ & $F_{\text {mean }}$ & $F_{\text {median }}$ & $F_{\text {worst }}$ & Std & \\
\hline $\mathrm{g} 01 *[4]$ & 85.0909 & 85.0909 & 85.0909 & 85.0909 & $1.2 \mathrm{e}-6$ & 85.0855 \\
\hline$g 02^{[7]}$ & 32 & 32 & 32 & 32 & 0 & 31.9998 \\
\hline$g 03^{[7]}$ & 28.25 & 28.25 & 28.25 & 28.25 & 0 & 31.2498 \\
\hline$g 04^{[8]}$ & 22.5000 & 22.5000 & 22.5000 & 22.5000 & 0 & 22.5006 \\
\hline$g 05^{[8]}$ & -26.000 & -25.9928 & -25.9988 & -25.9536 & 0.007 & -25.9297 \\
\hline$g 06^{[10,12,17]}$ & 225 & 225 & 225 & 225 & 0 & $225^{[10,12,17]}$ \\
\hline$g 07^{[10,13,17]}$ & $\mathbf{0}$ & $\mathbf{0}$ & $\mathbf{0}$ & $\mathbf{0}$ & $\mathbf{0}$ & $\begin{array}{c}0^{[10]}, 5^{[13]} \\
3.47^{[17]}\end{array}$ \\
\hline$g 08^{[10,17]}$ & -29.2000 & -29.2000 & -29.2000 & -29.2000 & $1.2 \mathrm{e}-8$ & $\begin{array}{c}-29.2^{[10]}, \\
-29.198^{[17]}\end{array}$ \\
\hline$g 09^{[10,15]}$ & 81.3279 & 81.3279 & 81.3279 & 81.3279 & 0 & $\begin{array}{c}81.3279^{[10]}, \\
82.33^{[15]}\end{array}$ \\
\hline$g 10^{[10,15,17]}$ & 100.0000 & 100.0000 & 100.0000 & 100.0000 & 0 & $\begin{array}{c}100.0001^{[10]}, \\
100.58^{[15]}, \\
100.2050^{[17]}\end{array}$ \\
\hline $\mathrm{g} 11^{[14]}$ & -1 & -1 & -1 & -1 & 0 & -1 \\
\hline$g 12^{[14]}$ & -2.25 & -2.25 & -2.25 & -2.25 & 0 & -2.25 \\
\hline$g 13^{[14]}$ & 17 & 17 & 17 & 17 & 0 & 17 \\
\hline$g 14^{[14]}$ & 88.7866 & 88.7866 & 88.7866 & 88.7866 & 0 & 88.79 \\
\hline$g 15$ & 100.0000 & 100.0000 & 100.0000 & 100.0000 & 0 & $N A$ \\
\hline$g 16$ & 50.1654 & 50.1654 & 50.1654 & 50.1654 & $1.1 \mathrm{e}-7$ & $N A$ \\
\hline$g 17$ & 50.1114 & 50.1114 & 50.1114 & 50.1114 & $2.1 \mathrm{e}-10$ & $N A$ \\
\hline$g 18$ & -29.2000 & -29.2000 & -29.2000 & -29.2000 & $5.6 \mathrm{e}-7$ & $N A$ \\
\hline$g 19$ & -44.3468 & -44.3468 & -44.3468 & -44.3468 & $1.8 \mathrm{e}-5$ & $N A$ \\
\hline$g 20$ & 44.3417 & 44.3417 & 44.3417 & 44.3417 & 0 & $N A$ \\
\hline
\end{tabular}

Table 2. Comparison of the solutions found by EABDS and the related algorithms in references, $\mathrm{g}_{\text {mean }}$ and MNI needed by EABDS.

\begin{tabular}{|c|c|c|c|}
\hline \multirow{2}{*}{ No. } & \multirow{2}{*}{$\mathrm{g}_{\text {mean }} / \mathrm{MNI}$} & \multicolumn{2}{|c|}{$\left(x^{*}, y^{*}\right)$} \\
\hline & & EABDS & Ref \\
\hline $\mathrm{g} 01^{[4]}$ & $40 / 1353$ & $(17.4545,10.9091)$ & $(17.4500,10,9080)$ \\
\hline $\mathrm{g} 02^{[7]}$ & $42 / 1421$ & $(2,6)$ & $(2.0002,5.9999)$ \\
\hline $\mathrm{g} 03^{[7]}$ & $24 / 823$ & $(0,0)$ & $(1.0071,1.0035)$ \\
\hline $\mathrm{g} 04^{[8]}$ & $21 / 799$ & $(1.5000,4.5000)$ & $(4.4922,1.5223)$ \\
\hline $\mathrm{g} 05^{[8]}$ & $35 / 1332$ & $(0,0.9,0,0.6,0.4)$ & $(0,0.8965,0,0.5977,0.3906)$ \\
\hline $\mathrm{g} 06^{[10,12,17]}$ & $45 / 1469$ & $(20,5,10,5)$ & $(20,5,10,5)^{[10,12,17]}$ \\
\hline $\mathrm{g} 07^{[10,13,17]}$ & $41 / 1401$ & $(0,0,-10,-10)$ & $\begin{array}{c}(0,30,-10,10)^{[10]} \\
(25,30,5,10)^{[13]} \\
(24.87,29.98,5,10.31)^{[17]}\end{array}$ \\
\hline $\mathrm{g} 08^{[10,17]}$ & $29 / 1021$ & $(0,0.9,0,0.6,0.4)$ & $\begin{array}{c}(0,0.9,0,0.6,0.4)^{[10]} \\
(0,0.9,0,0.6,0.3999)^{[17]}\end{array}$ \\
\hline $\mathrm{g} 09^{[10,15]}$ & 23/1089 & $(10.0164,0.8197)$ & $\begin{array}{c}(10,0164,0.8197)^{[10]} \\
(10.04,0.1629)^{[15]} \\
(10.000,10.000)^{[10]}\end{array}$ \\
\hline $\operatorname{g} 10^{[10,15,17]}$ & $38 / 1184$ & $(10,10)$ & $\begin{array}{c}(10.03,9.969)^{[15]} \\
(10.01,9.93)^{[17]}\end{array}$ \\
\hline $\mathrm{g} 11^{[14]}$ & $29 / 897$ & $(0.5,0.5,0.5,0.5)$ & NA \\
\hline $\mathrm{g} 12^{[14]}$ & $24 / 765$ & $(0.75,0.75,0.75,0.75)$ & NA \\
\hline $\mathrm{g} 13^{[14]}$ & $31 / 959$ & $(1,0)$ & NA \\
\hline $\mathrm{g} 14^{[14]}$ & $21 / 682$ & $(0,0.5773)$ & NA \\
\hline $\mathrm{g} 15$ & $46 / 1396$ & $(10,10)$ & NA \\
\hline g16 & $50 / 1528$ & $(5.2876,5.2876)$ & NA \\
\hline $\mathrm{g} 17$ & $35 / 1102$ & $(4.7640,4.7640)$ & NA \\
\hline g18 & $36 / 1125$ & $(0,0.9,0,0.6,0.4)$ & NA \\
\hline g19 & $36 / 1104$ & $(0.3192,0,1.2490,1.0498,1.1992)$ & NA \\
\hline g20 & $21 / 657$ & $(0,0,1.1903,1.1903,0.3805)$ & NA \\
\hline
\end{tabular}

vex follower's functions. For other problems except for $g 02$, the best results found by EABDS are almost as good as those by the compared algorithms. For $g 02$, although the result found by EABDS is a little bit worse than that by the compared algorithm, it should be noted that [7] provides an approximate solution of the follower's prob- 
lem, which makes the result better than that by EABDS, in fact, EABDS provides a precise solution. In all 30 runs, EABDS finds the best results of all problems except for problems $g 01, g 05, g 08$ and $g 16-g 19$. For these problems, from Table 1, we can find the worst results are close to the best results and the standard deviations are also very small. This means that EABDS is stable and robust.

From Table 2, we can also see that for all of the problems, EABDS needs much less MNI and $g_{\text {mean }}$, which means the proposed algorithm is efficient.

\section{Conclusions}

For the nonlinear bilevel programming problems with a special follower's objective, we propose an evolutionary algorithm based on a decomposition scheme, in which based on the best points generated so far, we design a new crossover operator, and apply the approximate SWIFT to deal with the leader's constraints. From the data simulation, we can also see easily that the proposed algorithm is especially effective and efficient for solving this kind of BLPPs.

\section{Acknowledgment}

This work was supported by the National Natural Science Foundation of China (No. 60873099).

\section{References}

[1] B. Colson, P. Marcotte, and G. Savard, "Bilevel programming: A survey," A Quarterly Journal of Operations Research (4OR), Vol. 3, pp. 87-107, 2005.

[2] J. F. Bard, "Practical bilevel optimization," The Netherlands: Kluwer Academic Publishers, 1998.

[3] U. P. Wen and S. T. Hsu, "Linear bi-level programming problems-A review," The Journal of the Operational Research Society, Vol. 42, pp. 125-133, 1991.

[4] K.-M. Lan, U.-P. Wen, H.-S. Shih, et al., "A hybrid neural network approach to bilevel programming problems," Applied Mathematics Letters, Vol. 20, pp. 880-884, 2007.

[5] H. I. Calvete, C. Gale, and P. M. Mateo, "A new approach for solving linear bilevel problems using genetic algorithms," European Journal of Operational Research, Vol. 188, pp. 14-28, 2008

[6] L. D. Muu and N. V. Quy, "A global optimization method for solving convex quadratic bilevel programming problems," Journal of Global Optimization, Vol. 26, pp. 199-219, 2003.
[7] D. L. Zhu, Q. Xua, and Z. H. Lin, "A homotopy methodfor solving bilevel programming problem," Nonlinear Analysis, Vol. 57, pp. 917-928, 2004.

[8] H. Tuy, A. Migdalas, and N. T. Hoai-Phuong, "A novel approach to bilevel nonlinear programming," Journal of Global Optimization, Vol. 38, pp. 527-554, 2007

[9] H. I. Calvete and C. Gale, "On the quasiconcave bilevel programming problem," Journal of Optimization Theory and Applications, Vol. 98, pp. 613-622, 1998.

[10] Y. P. Wang, Y. C. Jiao, and H. Li, “An evolutionary algorithm for solving nonlinear bilevel programming based on a new constraint - handling scheme," IEEE Trans. on Systems, Man, and Cybernetics-Part C, Vol. 35, pp. 221232,2005 .

[11] H. C. Li and Y. P. Wang, "A hybrid genetic algorithm for solving a class of nonlinear bilevel programming problems," Proceedings of Simulated Evolution and Learning6th International Conference, SEAL, pp. 408-415, 2006.

[12] K. Shimizu and E. Aiyoshi, "A new computational method for Stackelberg and minmax problems by use of a penalty method," IEEE Transactions on Automatic Control, Vol. 26, pp. 460-466, 1998.

[13] E. Aiyoshi and K. Shimizu, "A solution method for the static constrained Stackelberg problem via penalty method," IEEE Transactions on Automatic Control, Vol. AC-29 (12), pp. 1111-1114, 1984.

[14] B. Colson, P. Marcotte, and G. Savard, “A trust-region method for nonlinear bilevel programming: algorithm \& computational experience," Computational Optimization and Applications, Vol. 30, pp. 211-227, 2005.

[15] V. Oduguwa and R. Roy, "Bilevel optimization using genetic algorithm," Proceedings of IEEE International Conference on Artificial Intelligence Systems, pp. 123$128,2002$.

[16] B. D. Liu, "Stackelberg-Nash equilibrium for mutilevel programming with multiple followers using genetic algorithms," Computer mathematics Application, Vol. 36, No. 7, pp. 79-89, 1998.

[17] X. B. Zhu, Q. Yu, and X. J. Wang, "A hybrid differential evolution algorithm for solving nonlinear bilevel programming with linear constraints," Proceedings of 5th IEEE International Conference on Cognitive Informatics (ICCI'06), pp. 126-131, 2006.

[18] J. Rajesh, K. Gupta, and H. Shankar, et al., "A tabu search based approach for solving a class of bilevel programming problems in chemical engineering," Journal of Heuristics, Vol. 9, pp. 307-319, 2003.

[19] B. V. Sheela and P. Ramamoorthy, "SWIFT-S new constraines optimization technique," Computer Methods in Applied Mechanics and Engineering, Vol. 6, pp. 309-318, 1975. 